\title{
Contribuições e perspectivas da pesquisa brasileira sobre plantas alimentícias silvestres com foco no semiárido
}

\author{
Mirna Andrade Bezerra',* (D), José Machado Moita Neto' ${ }^{(0)}$, Ivanilza Moreira de Andrade ${ }^{2}(\mathbb{D}$, \\ Francisco Soares Santos Filho" (D)
}

\author{
'Universidade Federal do Piauí - UFPI, Teresina, Piauí, Brasil \\ ¿Universidade Federal Delta do Parnaíba - UFDPar, Parnaíba, Piauí, Brasil \\ 3Universidade Estadual do Piauí - UESPI, Teresina, Piauí, Brasil \\ *Autor para correspondência: mirna.a.bezl1@gmail.com
}

Submetido em 08.IX.2020

Aceito em 28.IX.2021

DOI 10.21826/2446-82312022v77e2022003

RESUMO - As plantas alimentícias silvestres (PAS) têm amplo valor para diversos povos do mundo para alcançar a segurança alimentar. No entanto, apesar da rica biodiversidade, no Brasil a pesquisa sobre este tema é incipiente. Desta forma, objetivou-se mapear os artigos científicos brasileiros publicados sobre PAS e identificar as famílias e espécies alimentícias em trabalhos etnobotânicos, especialmente para a região semiárida. Na revisão científica realizada neste artigo utilizou os seguintes descritores: plantas alimentícias, silvestres, Brasil e Nordeste nas bases de dados "Web of Science" e "Scopus". O levantamento em bases internacionais apontou o Brasil com 11 publicações, sugerindo que os estudos sobre PAS são desproporcionais à diversidade biológica. Destaca-se a necessidade de pesquisas futuras sobre a composição química e nutricional das espécies utilizadas pelas populações, valorização dos modos de vida que legitimaram o uso e aplicações das plantas.

Palavras-chave: etnobotânica, nordeste, plantas comestíveis silvestres, segurança alimentar.

ABSTRACT - Contributions and perspectives of Brazilian research on wild food plants with a focus on the semiarid region. Wild food plants (PAS) are of great value to different peoples in the world for achieving food security. However, despite the rich biodiversity, research on this topic in Brazil is incipient. Thus, the objective was to map the Brazilian scientific articles published on PAS and to identify families and food species in ethnobotanical works, especially for the semiarid region. The scientific review carried out in this article used the following descriptors: food plants, wild, Brazil and Northeast in the "Web of Science" and "Scopus" databases. The survey on an international basis pointed to Brazil with 11 publications, suggesting that studies on PAS are disproportionate to biological diversity. The need for future research on the chemical and nutritional composition of the species used by the populations is highlighted, valuing the ways of life that legitimized the use and applications of plants.

Keywords: ethnobotany, food safety, northeast, wild edible plants.

\section{INTRODUÇÃO}

A utilização e a relação estabelecida com as plantas estão direta e indiretamente relacionadas ao desenvolvimento dos seres humanos, bem como ao estabelecimento e à multiplicação de indivíduos nos territórios (Lins Neto \& Albuquerque 2018). A trajetória evolutiva humana conta com a disseminação deliberada de sementes selvagens, posterior seleção de variáveis genéticas, domesticação de plantas vantajosas e, por último, o desenvolvimento da agricultura, que permitiu a produção em maior quantidade em resposta ao crescimento populacional em todo o globo (Veasey et al. 2011).

Tomchinsky \& Ming (2019) em levantamento histórico dos séculos XVI e XVII sobre as plantas comestíveis no Brasil, partindo de documentos da época, revelam relativa diversidade de espécies para a alimentação, como a mandioca (Manihot esculenta Crantz), abacaxi [Ananas comosus (L.)
Merril], jenipapo (Genipa americana L.), batata-doce [Ipomoea batatas L. (Lam.)] e uma acelerada introdução de muitas espécies exóticas que hoje são amplamente utilizadas. No entanto, apesar de tantos anos decorridos, no Brasil e no mundo o suprimento alimentar das populações baseia-se em menos de 30 espécies, tornando o cultivo altamente especializado, em larga escala é responsável por exaurir recursos naturais (Barbieri et al. 2014).

A demanda econômica, nutricional e populacional motivou o consumo de espécies que eram reconhecidamente ricas em proteínas e carboidratos, de fácil colheita, curto período de desenvolvimento, selecionando um pequeno grupo de espécies inseridas na base alimentar (Raven et al. 2014), destinando outras espécies iguais ou superiores em qualidade, ao esquecimento, degradação ou extinção antes mesmo de serem conhecidas.

Especialmente no Brasil, onde há "níveis excepcionais de biodiversidade" (Martinelli \& Moraes 2013), estima-se 
que são reconhecidas 49.989 espécies para a flora brasileira, incluindo as nativas, cultivadas e naturalizadas (Flora do Brasil 2020, 2021). Dentre elas há uma variedade de espécies vegetais denominadas de plantas alimentícias silvestres com uso negligenciado (Nascimento et al. 2012). Kinupp \& Lorenzi (2014) incluem as plantas alimentícias silvestres dentro do espectro de Plantas Alimentícias Não Convencionais (PANC). Estas agregam plantas de uso não convencional (estão incluídas as plantas alimentícias silvestres) e partes de plantas (folhas, frutos, raízes etc.) de uso convencional, para as quais são relatados seus usos em estudos nacionais e internacionais.

Hora et al. (2020) delimitam e consideram as plantas silvestres como espécies não cultivadas, mas que podem ser coletadas em "ambientes naturais (incluindo ao redor das residências, rios, lagos, florestas, e até mesmo paisagens agrícolas)". Em contrapartida, Borelli et al. (2020), em um artigo de revisão, adotam o conceito de que "o termo 'plantas silvestres' é estendido a todas as plantas alimentícias (incluindo ervas e especiarias) que também são semi-domesticadas, além de produtos alimentícios florestais não madeireiros economicamente importantes". Para estes autores, as plantas alimentícias silvestres serão consideradas como espécies espontâneas coletadas em florestas, bosques, terrenos baldios, quintais, entre outros e classificadas, segundo Guinand \& Lemessa (2001), como produtos florestais não madeireiros - PFNMs.

As plantas alimentícias silvestres (PAS) são consideradas de amplo valor para diversos povos do mundo, para alcançar a segurança alimentar, assim como, por exemplo, nos resultados obtidos na África (sendo incluídos países como Nigéria, Gana, Etiópia, dentre outros quanto ao uso de hortaliças e frutos silvestres) relatados por Aworh (2018), no Peru por Cruz-Garcia et al. (2018) e na Turquia por Akgul et al. (2018). No Brasil, elas são conhecidas e utilizadas por populações tradicionais, comunidades rurais, ribeirinhas, dentre outras, para atender as necessidades básicas de alimentação e saúde (Cruz et al. 2013, Campos et al. 2015).

Em regiões com problemas de escassez de alimentos relacionados a fatores climáticos e econômicos, como em algumas áreas do nordeste brasileiro (Nascimento et al. 2012), são utilizadas espécies silvestres como solução para a fome. Aworh (2018) relata que na África essas plantas ainda são pouco conhecidas, entretanto, em países vulneráveis economicamente e com desigualdades sociais que impõe a busca por alimentos nas matas elas são bastante utilizadas pelas populações (Lulekal et al. 2011), são altamente adaptadas às condições severas de crescimento e prosperam com poucos cuidados. Da mesma forma, no Brasil, constatam-se em estudos de anatomia vegetal, as adaptações desenvolvidas a condições severas, como espessamento de cutícula, disposição de estômatos, presença de compostos que permitem seu desenvolvimento e consequente aproveitamento pelos seres vivos em períodos de escassez ou situações adversas para a sobrevivência (Barros \& Soares 2013).
O Nordeste brasileiro possui predominantemente a vegetação tropical semiárida característica da caatinga, que é exclusiva do Brasil (Andrade-Lima 1981). A precipitação anual é distribuída irregularmente no tempo e no espaço, a estação seca compreende de cinco a nove meses, gerando períodos de difícil sobrevivência para populações que vivem nestas áreas (Araújo et al. 2007). No entanto, a riqueza do extrato arbóreo é significativa, assim como a herbácea que ainda é pouco conhecida e estudada (Araújo et al. 2007), indicando possibilidades de usos variados.

Tendo em vista o potencial inexplorado do Nordeste e da caatinga, estudos estão sendo conduzidos em Pernambuco, Paraíba, Alagoas, Piauí, Rio Grande do Norte e outros estados desta região a fim de conhecer os usos e a diversidade da flora local, especialmente da região semiárida. No entanto, na contramão das evidências sobre o potencial destas plantas, Kinupp \& Barros (2008) e Bortolotto et al. (2017), em estudos realizados em regiões brasileiras distintas (Sul e Centro-Oeste, respectivamente) e há algum tempo, já relatam a subutilização de tais recursos e a perda dos conhecimentos relacionados aos usos tradicionais, ressaltando o valor dos estudos etnobotânicos para a identificação e documentação dos recursos alimentícios muitas vezes associados a contextos ecológicos locais e culturais diversificados.

O levantamento de artigos científicos sobre plantas de uso alimentício poderá contribuir na indicação de caminhos para pesquisas futuras, já que os trabalhos nesta área ainda são pouco explorados. Desta forma, objetivase mapear os artigos científicos que trazem pesquisas científicas desenvolvidas no Brasil publicadas sobre plantas alimentícias silvestres, com destaque às pesquisas desenvolvidas na região semiárida, além de identificar as principais famílias e espécies alimentícias apontadas por meio desta pesquisa.

\section{METODOLOGIA}

O levantamento de artigos científicos foi realizado em bases de dados eletrônicas no período de maio a junho de 2020. A fim de selecionar descritores que agregassem maior quantidade de artigos científicos, foram testadas palavraschave relacionadas às plantas alimentícias silvestres como plantas comestíveis silvestres, plantas alimentícias nativas, dentre outros termos relacionados. No entanto, ao final desta primeira seleção os seguintes termos e as concatenações entre estes foram as que mais proporcionaram amplitude de dados: plantas alimentícias ("Food Plants"), silvestres ("Wild"), Brasil ("Brazil") e nordestino ("Norteastern").

A pesquisa para coleta e quantificação dos trabalhos foi realizada nas bases de dados Web of Science (2020) e Scopus (2020). As palavras foram inseridas no campo de busca das bases com as seguintes associações: "Food Plants"; "Food Plants" AND "Wild"; "Food Plants"AND "Wild"AND "Brazil"; "Food Plants"AND "Wild" AND "Brazil" AND "Norteastern". 
Os trabalhos identificados apresentaram os descritores selecionados no título, resumo e/ou palavras-chave e não foi utilizada uma delimitação temporal, exceto a estabelecida pelos próprios limites da base de dados, focando a análise no período em que as publicações nesta área passaram a ser divulgadas. Foram excluídos artigos científicos e estudos que estavam em duplicidade nas bases de dados, que não foram desenvolvidos no Brasil, os que não eram etnobotânicos e/ou não se tratavam de pesquisas com plantas alimentícias de ocorrência espontânea (PAS).

As pesquisas realizadas no Brasil foram identificadas e quantificadas quanto à distribuição geográfica das áreas estudadas. Assim como, uma lista de espécies alimentícias citadas pelos autores nos resultados dos artigos e as principais famílias botânicas estão elencadas em tabelas e gráficos. As espécies citadas nos artigos científicos foram analisadas quanto à nomenclatura atual e uma triagem foi realizada a partir dos dados disponibilizados no sitio eletrônico da Flora do Brasil 2020 (2021).

A busca com os descritores "Food Plants"; "Food Plants" AND "Wild" proporcionaram um comparativo entre estudos brasileiros e internacionais, áreas de pesquisa e análise de distribuição temporal das produções científicas. Os dados coletados foram processados em planilhas eletrônicas do software Microsoft Excel 2010.

\section{RESULTADOS E DISCUSSÃO}

\section{Análise quantitativa de artigos científicos sobre PAS realizadas no Brasil}

As bases de dados "WoS" e "Scopus, são responsáveis por reunir grande quantidade de trabalhos do mundo todo. Nas buscas realizadas, apenas 11 trabalhos de estudos realizados no Brasil foram identificados utilizando as palavras-chave "Food Plants" AND "Wild" AND "Brazil" (Tab. 1).

Nesta primeira análise sobre o quantitativo de trabalhos científicos, observa-se uma lacuna quanto ao desenvolvimento de pesquisas em uma área tão relevante para o conhecimento da biodiversidade brasileira. Nascimento et al. (2011) ressaltam que na caatinga muitas espécies são importantes e fundamentais para a sobrevivência de grupos humanos, mas apenas nos últimos anos essa relação foi mais estudada, principalmente para fins medicinais. Liporacci et al. (2017) confirmam em uma revisão sistemática que há regiões na caatinga e também na mata atlântica que ainda precisam ser mais estudadas, pois possuem formações vegetais heterogêneas e que carecem de estudos etnobotânicos.
O conhecimento das espécies está intimamente relacionado com a cultura local. A conservação dos recursos genéticos contidos nestas plantas pode ser resguardada pelas comunidades. Entretanto, precisam ser reconhecidas, reintroduzidas nos hábitos alimentares das comunidades que em alguns casos associam o uso com tempos difíceis e repudiam o seu uso. Alternativamente, estratégias de consumo sustentável com amparo adequado de políticas públicas podem gerar renda, reaproximá-los da cultura de uso de espécies nativas e promover a proteção aos recursos naturais das comunidades. (Bortolotto et al. 2017, Bortolotto et al. 2019).

$\mathrm{O}$ reduzido e/ou recente interesse pela temática torna também vulnerável os conhecimentos e saberes tradicionais guardados, na grande maioria das vezes por pessoas mais experientes no contato com as plantas, em comunidades tradicionais ou não e que aos poucos se perdem por inúmeros motivos, sejam pela falta de interesse dos mais jovens, pelos avanços tecnológicos ou pela saída do ambiente rural para o ambiente urbano (Chaves et al. 2017). O desligamento e/ou interrupção do fluxo destes saberes põe em risco os recursos naturais e a sua conservação (Bortolotto et al. 2017).

As publicações brasileiras, em ambas as bases ("WoS" e "Scopus"), possuem a mesma quantidade de publicações, totalizando 11 trabalhos, dos quais se constatou que seis artigos eram comuns entre as bases ou não estavam relacionados às plantas alimentícias silvestres. Partindo disso, a Tab. 2 evidencia as pesquisas que podem ser encontradas nas bases que foram utilizadas para este estudo.

Apesar de poucas pesquisas sobre plantas alimentícias silvestres no Brasil, o Nordeste apresenta alta representatividade. A maioria das pesquisas foi desenvolvida, principalmente nesta região: Pernambuco (quatro), Paraíba (um), Piauí (um), Alagoas (um) e Ceará (um).

A Fig. 1 elenca e demonstra a distribuição geográfica dos estudos mencionados, revelando vastas possibilidades de análise, documentação de saberes, formas de uso, relação homem-natureza, critérios de escolha para utilização doméstica ou comercial sobre plantas alimentícias silvestres.

$\mathrm{O}$ estado de Pernambuco possui a maior quantidade de estudos relacionados às plantas alimentícias silvestres e plantas medicinais, aliadas ao conhecimento e uso por parte de populações consideradas tradicionais ou não (Ritter et al., 2015; Liporacci et al., 2017). A etnobotânica é utilizada na maioria dos estudos selecionados como uma ciência fundamental e com metodologias que permitem o acesso aos conhecimentos sobre os recursos naturais e a cultura local associada.

Tabela 1. Descritores e número de trabalhos depositados nas bases de dados Web of Science e Scopus.

\begin{tabular}{lcc}
\hline Descritores & Web of Science & Scopus \\
\hline "Food Plants" AND "Wild" & 480 & 541 \\
"Food Plants" AND "Wild" AND "Brazil" & 11 & 11 \\
"Food Plants" AND "Wild" AND "Brazil” AND "Northeastern" & 5 & 3 \\
\hline
\end{tabular}


Tabela 2. Artigos científicos sobre Plantas Alimentícias Silvestres, realizados no Brasil, encontrados nas bases Web of Science e Scopus.

\begin{tabular}{|c|c|c|c|}
\hline Título & Autor (ano) & Área de estudo & Periódico \\
\hline $\begin{array}{l}\text { Protein and mineral contents of native species, } \\
\text { potential vegetables, and fruits }\end{array}$ & Kinupp \& Barros (2008) & Rio Grande do Sul & $\begin{array}{l}\text { Ciência e Tecnologia de } \\
\text { Alimentos }\end{array}$ \\
\hline $\begin{array}{l}\text { Chemical characterization of native wild plants of dry } \\
\text { seasonal forests of the semi-arid region of northeastern } \\
\text { Brazil }\end{array}$ & Nascimento et al. (2011) & Pernambuco e Paraíba & Food Research International \\
\hline $\begin{array}{l}\text { Knowledge, use and management of native wild edible } \\
\text { plants from a seasonal dry forest (NE, Brazil) }\end{array}$ & Cruz et al.(2013) & Pernambuco & $\begin{array}{l}\text { Journal of Ethnobiology } \\
\text { and Ethnomedicine }\end{array}$ \\
\hline $\begin{array}{l}\text { Knowledge and use of wild food plants in areas of dry } \\
\text { seasonal forests in Brazil }\end{array}$ & Nascimento et al.(2013) & Pernambuco & $\begin{array}{l}\text { Ecology of Food and } \\
\text { Nutrition }\end{array}$ \\
\hline $\begin{array}{l}\text { "I eat the manofê so it is not forgotten": local } \\
\text { perceptions and consumption of native wild edible } \\
\text { plants from seasonal dry forests in Brazil }\end{array}$ & Cruz et al.(2014) & Pernambuco & $\begin{array}{l}\text { Journal of Ethnobiology } \\
\text { and Ethnomedicine }\end{array}$ \\
\hline $\begin{array}{l}\text { Potential of wild food plants from the semi-arid region } \\
\text { of Northeast Brazil: chemical approach ethnoguided }\end{array}$ & Chaves et al. (2015) & Piauí & Espacios \\
\hline $\begin{array}{l}\text { Do socioeconomic characteristics explain the } \\
\text { knowledge and use of native food plants in semiarid } \\
\text { environments in Northeastern Brazil? }\end{array}$ & Campos et al. (2015) & Ceará & Journal of Arid Environments \\
\hline $\begin{array}{l}\text { A knowledge network to promote the use and } \\
\text { valorization of wild food plants in the Pantanal and } \\
\text { Cerrado, Brazil }\end{array}$ & Bortolotto et al.(2017) & Mato Grosso do Sul & $\begin{array}{l}\text { Regional Environmental } \\
\text { Change }\end{array}$ \\
\hline $\begin{array}{l}\text { Preliminary list of native food plants of Mato Grosso } \\
\text { do Sul, Brazil }\end{array}$ & Bortolotto et al.(2018) & Mato Grosso do Sul & Iheringia - série Botânica \\
\hline $\begin{array}{l}\text { Local knowledge about native food plants in Brazilian } \\
\text { chaco }\end{array}$ & Bortolotto et al.(2019) & Mato Grosso do Sul & Oecologia Australis \\
\hline $\begin{array}{l}\text { Local criteria for the selection of wild food plants for } \\
\text { consumption and sale in Alagoas, Brazil }\end{array}$ & Gomes et al.(2020) & Alagoas & $\begin{array}{l}\text { Ethnobiology and } \\
\text { Conservation }\end{array}$ \\
\hline
\end{tabular}

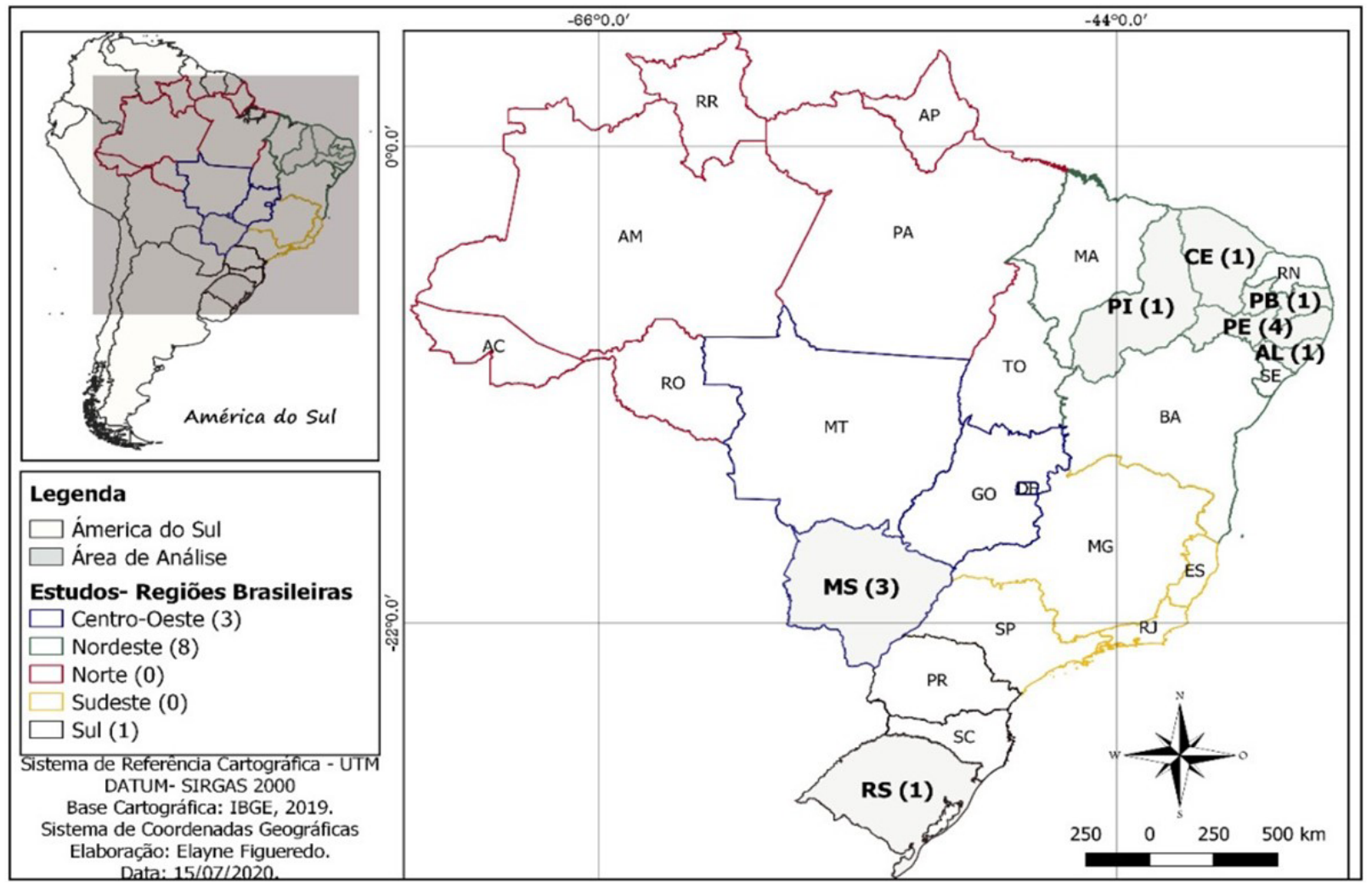

Figura 1. Estudos realizados no Brasil sobre plantas alimentícias silvestres, encontrados nas bases de dados Web of Science e Scopus. 
Vale ainda ressaltar que a devolução de resultados de pesquisas etnobotânicas às comunidades possibilita a aproximação dos saberes das populações aos conhecimentos científicos e vice e versa. Esta conexão viabiliza o conhecimento e conservação mesmo que tardio (em alguns casos) da biodiversidade brasileira pelas próprias comunidades beneficiadas pela variedade de plantas silvestres existentes em sua localidade.

O estudo mais recente sobre plantas alimentícias silvestres foi realizado em Alagoas em duas comunidades do município de Piaçabuçu (Gomes et al. 2020), a fim de identificar o critério de seleção das espécies vegetais para o consumo, ressaltando a grande importância e implicações de tais ações para a conservação biológica.

Foram registrados três artigos desenvolvidos em Mato Grosso do Sul em regiões do Pantanal e do Cerrado (Bortolotto et al. 2017) e também na vegetação do tipo Chaco (Bortolotto et al. 2017) encontrado somente em alguns países da América do Sul como Argentina, Paraguai, Bolívia e Brasil. Destacam-se estas pesquisas, pois exceto os trabalhos realizados em estados nordestinos, o estado de Mato Grosso do Sul é o segundo em quantidade de publicações encontradas com os critérios adotados neste trabalho.

O estado do Piauí também possui uma publicação na base de dados sobre plantas alimentícias silvestres. No entanto, vários são os trabalhos desenvolvidos com plantas medicinais (Oliveira \& Barros 2018, Cunha \& Barros 2018, Farias et al. 2019, dentre outros) que também podem ter contribuições relevantes na identificação e uso de recursos alimentícios. Ao longo de muitos anos a busca por plantas para alimentação pode indicar que espécies foram selecionadas para fins medicinais a partir de propriedades quimiossensoriais como gosto amargo ou cheiro, permitindo associações para usos medicinais (Albuquerque et al. 2020) e/ou alimentícios.

De forma geral, as publicações estão distribuídas entre os anos de 2008 e 2020, sendo que em 2013 ocorreram duas publicações. O periódico com maior quantidade de publicação é o "Journal of Ethnobiology and Ethnomedicine", juntamente com Ethnobiology and Conservation reforçam a importância da Etnobotânica para a realização de estudos sobre plantas alimentícias silvestres. Esta observação não invalida a contribuição dos outros periódicos, pois tratam de áreas de pesquisa de igual relevância como a ecologia, a botânica e a conservação de ecossistemas fundamentais a manutenção dos recursos genéticos tão ameaçados pelos avanços da urbanização e do desenvolvimento econômico.

\section{A produção científica sobre PAS: comparativo, áreas de pesquisa e distribuição temporal}

$\mathrm{Na}$ base de dados Web of Science a maior quantidade de publicações está relacionada ao estudo das plantas (238). No entanto, apresentam quantidades expressivas as áreas de pesquisa da farmácia/farmacologia (100), conservação da biodiversidade (76), Ciências ambientais/ ecologia (60), entre outras que estão elencadas na Fig. 2. Relaciona-se o alto quantitativo de trabalhos na área de farmácia/farmacologia ao estudo bem consolidado de plantas medicinais que também podem ser utilizadas como alimentícias por populações tradicionais no seu cotidiano em diversos países.

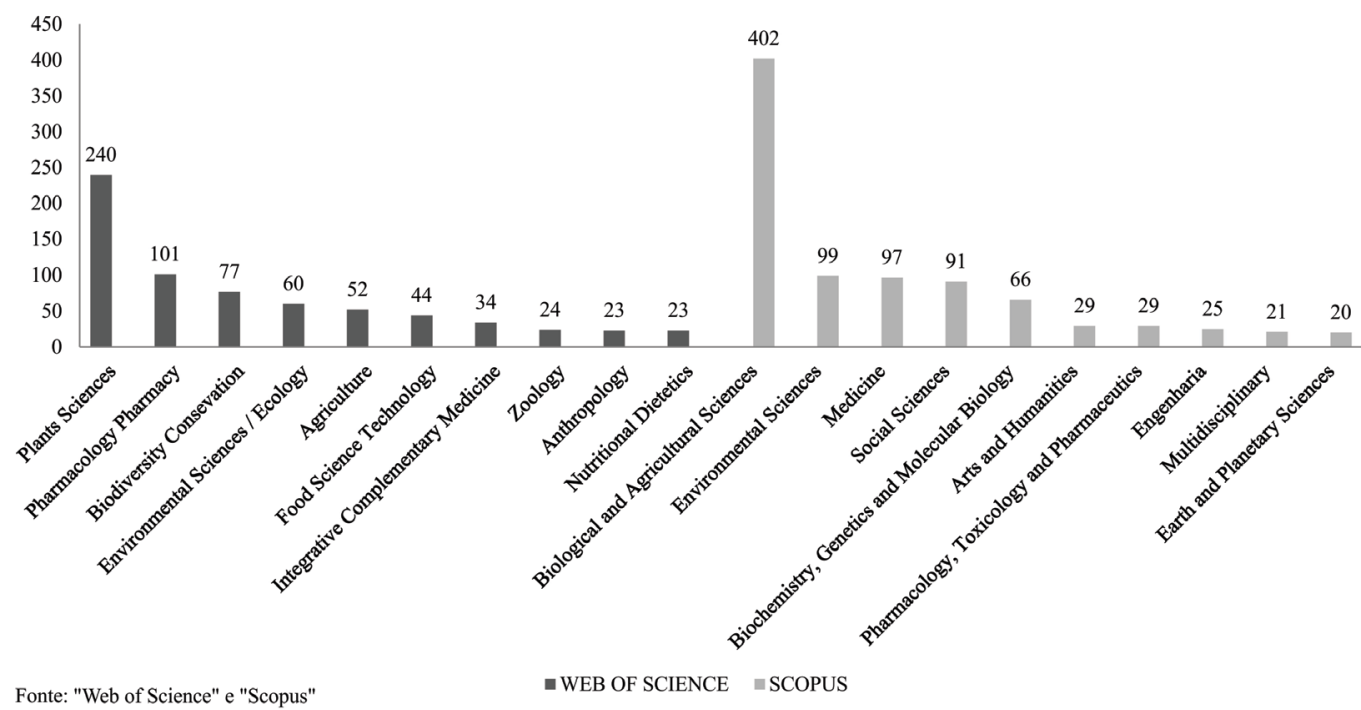

Figura 2. Número de publicações por área de pesquisa nas bases de dados "Web of Science" e "Scopus", utilizando-se os descritores "Food Plants" AND "Wild". 
A base de dados "Scopus" elenca outras áreas de pesquisa e em comparação com a "Web of Science" que se caracterizam por serem mais gerais. Os descritores utilizados apontam a grande área do conhecimento ciências biológicas e agrárias com o número mais elevado de publicações na temática em questão, com o total de 398 estudos. As ciências ambientais também aparecem com um número relevante de publicações, assim como medicina.

Outro dado importante coletado com os descritores "Food Plants" AND "Wild" foram os anos que mais apresentaram publicações. A base "WoS" obteve maior número no ano de 2019 com 49 trabalhos e a "Scopus" no ano de 2017 com 41, seguido por 2019 com 39 trabalhos (Fig. 3).

Para esta pesquisa não foi aplicada uma delimitação temporal e a Fig. 3 mostra os trabalhos a partir do ano 1996, dado que neste ano houve um aumento das publicações, apesar da baixa verificada em 1998 para ambas as bases houve significativo aumento nos anos posteriores. Desta forma, a primeira publicação sobre plantas alimentícias silvestres (considerando os descritores utilizados) foi verificada no ano de 1980, intitulada "The Utilization of Wild Food Plants by the Suiei Dorobo in Northern Kenya" pelo pesquisador Mitsuo Ichikawa da Universidade Kyoto (Ichikawa 1980)

Já na base "Scopus" o primeiro trabalho sobre plantas alimentícias silvestres teve registro para o ano de 1952, intitulado "Supplementary and emergency food plants of West Africa", no qual aponta a utilização destas plantas em situações de emergência como alternativa à quantidade insuficiente de alimentos para as populações (Irvine, 1952). A prospecção científica demonstrou que mesmo em nível mundial as plantas alimentícias são pouco estudadas.

Dentre os artigos científicos analisados foi possível observar e estabelecer um comparativo quanto à produção científica internacional e a nacional. Ambas as bases, apontaram Estados Unidos e Itália como os países que mais publicam em temáticas associadas ao conhecimento da biodiversidade de plantas alimentícias. Nascimento et al. (2013) elencam estudos recentes realizados na Patagônia nas regiões Andinas, extremo sul da América do Sul, que pretendem identificar o potencial alimentar e compreender os fatores que influenciam o conhecimento de comunidades rurais sobre recursos silvestres.

Em países do continente Africano, que possuem problemas sérios relacionados à fome e escassez de alimentos, estudos relacionados às plantas alimentícias silvestres e a viabilidade de segurança alimentar e nutricional também são expressivos, como ressaltado por Nascimento et al. (2012), há um crescente grupo de cientistas que se dedicam a examinar alimentos chamados emergenciais ou de fome, no qual as "abordagens variam desde a diversidade de plantas ao valor nutricional das espécies".

Apesar da relevância dos estudos realizados em países da América do Sul e na África, foram contabilizados para este último apenas 14 artigos. Nesta mesma análise, Colômbia e Brasil são os dois únicos países latinos com mais de dez trabalhos científicos levando em consideração a metodologia utilizada.

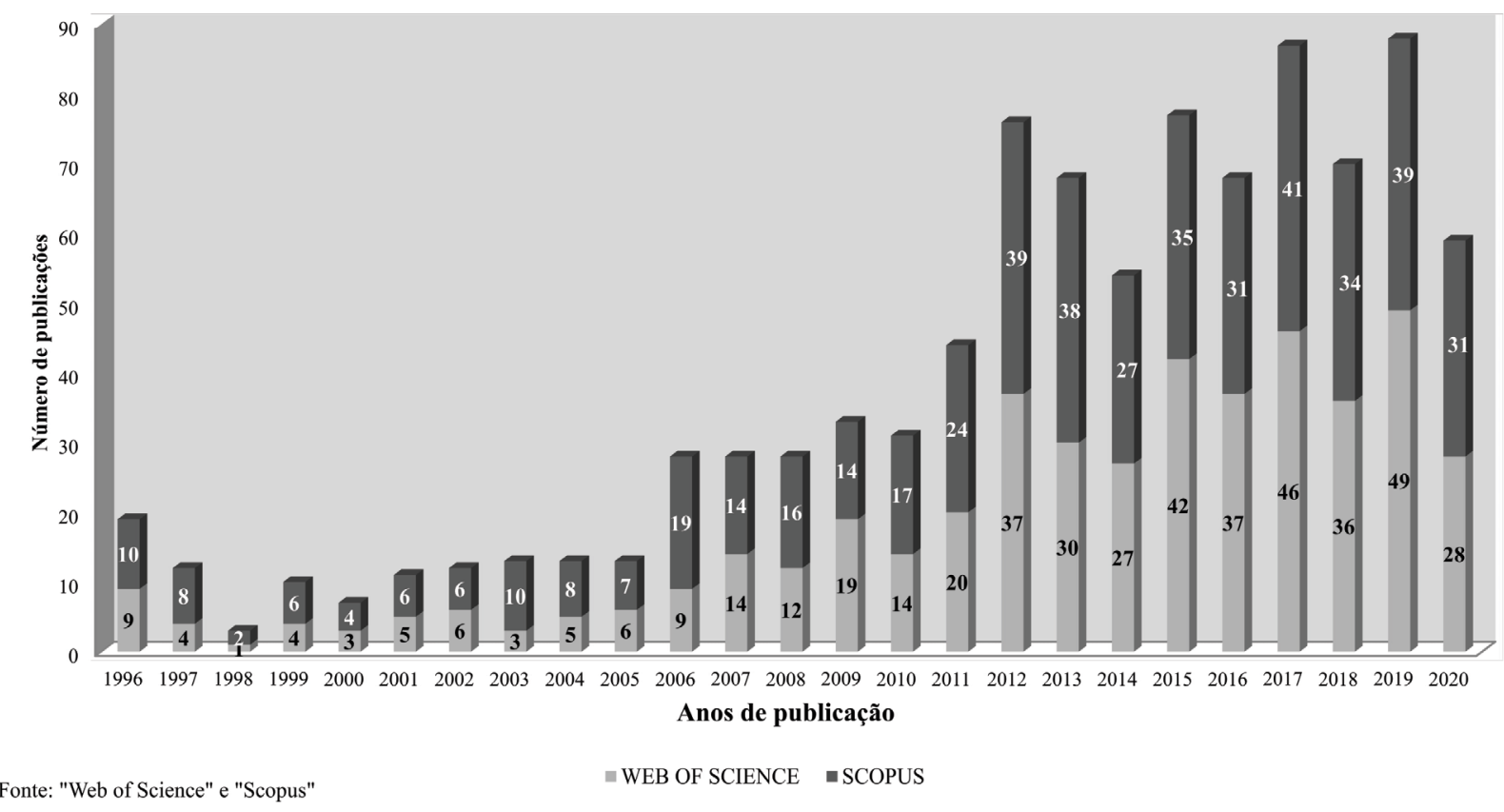

Figura 3. Número de publicações por ano nas bases de dados "Web of Science" e "Scopus", "Food Plants" AND "Wild" no período de 1996 a 2020. 


\section{Biodiversidade alimentícia identificada}

O conhecimento proporcionado pelos estudos científicos poderá permitir a proteção aos recursos naturais, às comunidades e aos seus saberes. Diante da relevância das pesquisas realizadas em grande parte na região Nordeste foi contabilizado dentre os trabalhos brasileiros, o total de oito investigações que apontaram as relações entre seres humanos e a natureza, os hábitos, escolhas e os critérios de seleção de espécies que ao longo do tempo foram fazendo parte da alimentação.

A quantidade de estudos desenvolvidos na região Nordeste pode estar relacionada à reconhecida problemática da seca, escassez de alimentos e população carente, motivando pesquisas que ampliem as possibilidades e identificação de alimentos validados pelas populações em situações emergenciais como sugerido por Nascimento et al. (2011, 2012, 2013), Campos et al. (2015) e Cruz et al. (2013). Além disso, há uma maior concentração de pesquisadores nesta região desenvolvendo estudos em etnobiologia, interessados na temática.

Toda a região Nordeste, especialmente a porção territorial com vegetação típica da caatinga, é marcada por uma curta estação chuvosa que permanece durante alguns meses e prolongado período de estiagem com consequências negativas para a colheita, desemprego em massa e perdas de economia (Nascimento et al. 2012, Jacob et al. 2020).

Entretanto, as consideráveis adversidades ambientais não interferem na variedade de plantas alimentícias que podem e são utilizadas pelas populações, propiciando benefícios sociais e econômicos, como por exemplo, nas comunidades extrativistas do estado do Alagoas que fazem uso doméstico e selecionam algumas espécies para comercialização e geração de renda (Gomes et al. 2020).

A Tab. 3 mostra um levantamento das espécies elencadas pelos pesquisadores em cinco estados brasileiros da região nordeste. Os artigos científicos foram referenciados e os nomes científicos das espécies foram devidamente conferidos e atualizados com base em pesquisas na Flora do Brasil 2020 (2021). Os estudos retratam uma pequena parcela da biodiversidade de espécies alimentícias, em sua grande maioria utilizada apenas localmente sem um valor adequado aos benefícios que proporcionam.

Ao todo, 90 espécies e 24 famílias foram citadas como alimentícias nestes trabalhos científicos. Além disso, é possível afirmar que a diversidade é ainda mais ampla, pois alguns trabalhos possuem a mesma área de estudo ou comunidades rurais como os trabalhos realizados por Nascimento et al. (2011), Nascimento et al. (2013), Cruz et al. (2013) e Cruz et al. (2014).

Algumas espécies citadas dentre os trabalhos analisados foram o araçá (Psidium spp.), trapiá (Crateva tapia L.), facheiro (Pilosocereus pachicladus F. Ritter.), mangaba (Hancornia speciosa Gomes), jatobá (Hymenaea courbaril L.), macambira (Bromelia laciniosa Mart. ex Schult. \& Schult. f.), coco catolé (Syagrus cearensis Noblick), ingá (Inga edulis Mart. e Inga laurina (Sw.) Willd.), dentre outras espécies com a utilização de diversas partes da planta como as raízes, as folhas, frutos, raízes, bem como uma variedade extensa de preparações que vão desde o seu uso isolado como em associação com plantas domesticadas e amplamente utilizadas.

Tabela 3. Família, espécie e nome popular de plantas alimentícias silvestres citadas em trabalhos realizados na região Nordeste do Brasil.

\begin{tabular}{|c|c|c|}
\hline Família/espécie & Nome popular & Fonte* \\
\hline \multicolumn{3}{|l|}{ ANACARDIACEAE } \\
\hline Schinus terebinthifolia Raddi & Aroeira & Gomes et al. 2020 \\
\hline Spondias sp. & Cajarana & Gomes et al. 2020 \\
\hline Spondias tuberosa Arruda & Imbú & Cruz et al. 2013, Nascimento et al. 2013, Cruz, et al. 2014 \\
\hline \multicolumn{3}{|l|}{ ANNONACEAE } \\
\hline Annona coriacea Mart. & Araticum & Campos et al. 2015 \\
\hline Duguetia furfuracea (A. St.-Hil.) Saff. & Pinha-brava & Campos et al. 2015 \\
\hline \multicolumn{3}{|l|}{ APOCYNACEAE } \\
\hline Hancornia speciosa & Mangaba & Campos et al. 2015; Gomes et al. 2020 \\
\hline Mandevilla tenuifolia (J. C. Mikan) Woodson & Manofê & $\begin{array}{c}\text { Cruz et al. } 2013 \\
\text { Nascimento et al. 2013, Cruz et al. } 2014\end{array}$ \\
\hline \multicolumn{3}{|l|}{ ARECACEAE } \\
\hline Acrocomia aculeata (Jacq.) Lodd. ex Mart & Macaúba/ Macaíba & $\begin{array}{c}\text { Nascimento et al. 2011, Cruz et al. 2013, Nascimento et al. 2013, } \\
\text { Cruz et al. 2014, Campos et al. } 2015\end{array}$ \\
\hline Attalea funifera Mart. & Coco-piaçaba & Campos et al. 2015, Gomes et al. 2020 \\
\hline Attalea speciosa Mart. ex Spreng & Babaçu & Campos et al. 2015 \\
\hline Syagrus cearensis & Coco-catolé & $\begin{array}{l}\text { Nascimento et al. 2011, Cruz et al. } 2013 \\
\text { Nascimento et al. 2013, Cruz et al. 2014, Campos et al. } 2015\end{array}$ \\
\hline Syagrus coronata (Mart.) Becc. & Coco-ouricuri & Gomes et al. 2020 \\
\hline
\end{tabular}


Tabela 3. Cont.

\begin{tabular}{|c|c|c|}
\hline Família/espécie & Nome popular & Fonte* \\
\hline \multicolumn{3}{|l|}{ BORAGINACEAE } \\
\hline Varronia globosa Jacq. & Moleque-duro & Nascimento et al. 2013 \\
\hline \multicolumn{3}{|l|}{ Bromeliaceae } \\
\hline Bromelia laciniosa & Macambira-roxa & Nascimento et al. 2013, Chaves et al. 2015 \\
\hline $\begin{array}{l}\text { Encholirium spectabile Mart. ex Schult. \& } \\
\text { Schult. f. }\end{array}$ & Macambira & Nascimento et al. 2013 \\
\hline \multicolumn{3}{|l|}{ CACTACEAE } \\
\hline Cereus jamacaru & Mandacaru & $\begin{array}{c}\text { Nascimento et al. 2011, Cruz, et al. 2013, Nascimento et al. 2013, } \\
\text { Cruz, et al. 2014, Nascimento et al. } 2013\end{array}$ \\
\hline $\begin{array}{l}\text { Melocactus zehntneri (Britton \& Rose) } \\
\text { Luetzelb }\end{array}$ & Coroa-de-frade & Nascimento et al. 2013, Chaves et al. 2015 \\
\hline Pilosocereus pachicladus & Facheiro & $\begin{array}{c}\text { Nascimento et al. 2011, Cruz et al. 2013, Nascimento et al. 2013, } \\
\text { Cruz et al. } 2014\end{array}$ \\
\hline $\begin{array}{l}\text { Tacinga inamoena (K. Schum.) N. P. Taylor } \\
\text { \& Stuppy }\end{array}$ & Cumbeba & Nascimento et al. 2013, Cruz et al. 2014 \\
\hline Xiquexique gounellei & Xiquexique & Nascimento et al. 2013, Cruz et al. 2014 \\
\hline \multicolumn{3}{|l|}{ CAPPARACEAE } \\
\hline Crateva tapia & Trapiá & Nascimento et al. 2013, Cruz et al. 2014 \\
\hline Cynophalla flexuosa (L.) J. Presl & Feijão-de-boi & Nascimento et al. 2013 \\
\hline $\begin{array}{l}\text { Neocalyptrocalyx longifolium (Mart.) Cornejo } \\
\text { \& Iltis }\end{array}$ & Incó & Cruz et al. 2013, Nascimento et al. 2013, Cruz et al. 2014 \\
\hline \multicolumn{3}{|l|}{ CARYOCARACEAE } \\
\hline Caryocar coriaceum Wittm. & Pequi & Campos et al. 2015 \\
\hline \multicolumn{3}{|l|}{ CELASTRACEAE } \\
\hline Monteverdia rigida (Mart.) Biral & Bom-nome & Nascimento et al. 2013 \\
\hline \multicolumn{3}{|l|}{ CHRYSOBALANACEAE } \\
\hline Chrysobalanus icaco L. & Gagiru & Gomes et al. 2020 \\
\hline Moquilea tomentosa Benth. & Goiti & Gomes et al. 2020 \\
\hline \multicolumn{3}{|l|}{ DIOSCOREACEAE } \\
\hline Dioscorea coronata Hauman & Cará, inhame-do-mato & Nascimento et al. 2013 \\
\hline \multicolumn{3}{|l|}{ EUPHORBIACEAE } \\
\hline Cnidoscolus urens (L.) Arthur & Urtiga & Nascimento et al. 2013 \\
\hline Manihot dichotoma Ule. & Maniçoba & Nascimento et al. 2013 \\
\hline Manihot glaziovii Müll. Arg. & Purnunça & Nascimento et al. 2013 \\
\hline \multicolumn{3}{|l|}{ FABACEAE } \\
\hline Bauhinia cheilantha (Bong.) Steud. & Mororó & Nascimento et al. 2013 \\
\hline Hymenaea courbaril & Jatobá-mirim & $\begin{array}{c}\text { Cruz et al. 2013, Nascimento et al. 2013, Cruz et al. 2014, } \\
\text { Campos et al. } 2015\end{array}$ \\
\hline Hymenaea martiana & Jatobá & Chaves et al. 2015 \\
\hline Hymenaea sp. & Jatobá-de-cavalo & Campos et al. 2015 \\
\hline Hymenaea stignocarpa Mart. ex Hayne & Jatobá-de-veado & Campos et al. 2015 \\
\hline Inga edulis & Ingá & Nascimento et al. 2013 \\
\hline $\begin{array}{l}\text { Macropsychanthus grandiflorus (Mart. ex } \\
\text { Benth.) L.P. Queiroz \& Snak }\end{array}$ & Mucunã & Campos et al. 2015 \\
\hline $\begin{array}{l}\text { Senegalia bahiensis (Benth.) Seigler \& } \\
\text { Ebinger }\end{array}$ & Espinheiro & Nascimento et al. 2013 \\
\hline Swartzia flaemingii Raddi & Jacarandá & Chaves et al. 2015 \\
\hline \multicolumn{3}{|l|}{ MALPIGHIACEAE } \\
\hline Byrsonima sericea DC. & Murici-verdadeiro & Campos et al. 2015 \\
\hline
\end{tabular}


Tabela 3. Cont

\begin{tabular}{|c|c|c|}
\hline Família/espécie & Nome popular & Fonte* \\
\hline \multicolumn{3}{|l|}{ MARANTHACEAE } \\
\hline Maranta divaricata Roscoe & Cana-de-macaco & Cruz et al. 2013, Nascimento et al. 2013, Cruz et al. 2014 \\
\hline \multicolumn{3}{|l|}{ MELASTOMATACEAE } \\
\hline Mouriri pusa Gardner & Puçá & Campos et al. 2015 \\
\hline \multicolumn{3}{|l|}{ MYRTACEAE } \\
\hline Eugenia sp. & Fruta-bola & Campos et al. 2015 \\
\hline Eugenia punicifolia (Kunth) DC. & Aperta-cú & Campos et al. 2015 \\
\hline Eugenia pyriformis Cambess & Ubaia & Cruz et al. 2013, Nascimento et al. 2013, Cruz et al. 2014 \\
\hline $\begin{array}{l}\text { Myrciaria floribunda (H. West ex Wild.) O. } \\
\text { Berg }\end{array}$ & Cambuí & Campos et al. 2015, Gomes et al. 2020 \\
\hline Plinia phitrantha (Kiaersk.) Sobral & Batinga & $\begin{array}{l}\text { Cruz et al. 2013, Nascimento et al. 2013, Cruz et al. 2014, } \\
\text { Nascimento et al. } 2011\end{array}$ \\
\hline Psidium guajava L. & Goiaba & Nascimento et al. 2013 \\
\hline Psidium guineense Sw. & Araçá-verdadeiro & Campos et al. 2015 \\
\hline Psidium laruotteanum Cambess. & Araçá-preto & Campos et al. 2015 \\
\hline Psidium myrsinites DC. & Araçá-vermelho & Campos et al. 2015 \\
\hline Psidium schenckianum Kiaersk & Pirim & $\begin{array}{c}\text { Nascimento et al. 2011, Cruz et al. 2013; Nascimento et al. 2013, } \\
\text { Cruz et al. } 2014\end{array}$ \\
\hline Psidium sobraleanum Proença \& Landrum & Goiabinha & Campos et al. 2015 \\
\hline Psidium sp. & Araçá-amarelo & Campos et al. 2015 \\
\hline \multicolumn{3}{|l|}{ PASSIFLORACEAE } \\
\hline Passiflora cincinnata Mast. & Maracujá-boi & Nascimento et al. 2013, Campos et al. 2015 \\
\hline Passiflora edulis Sims. & Maracujá & Nascimento et al. 2013 \\
\hline Passiflora mucronata Lam. & Maracujá-de-estralo & Campos et al. 2015 \\
\hline Passiflora silvestris Vell & Maracujá-peroba & Campos et al. 2015 \\
\hline \multicolumn{3}{|l|}{ RHAMNACEAE } \\
\hline Ziziphus joazeiro & Juá & Nascimento et al. 2011, Nascimento et al. 2013 \\
\hline \multicolumn{3}{|l|}{ RUBIACEAE } \\
\hline Genipa americana $\mathrm{L}$. & Genipapo & Gomes et al. 2020 \\
\hline Randia armata (Sw.) DC & Taturapé & Chaves et al. 2015 \\
\hline $\begin{array}{l}\text { Tocoyena formosa (Cham. \& Schltdl.) K. } \\
\text { Schum }\end{array}$ & Jenipapo & Campos et al. 2015 \\
\hline \multicolumn{3}{|l|}{ SAPINDACEAE } \\
\hline Talisia esculenta (Cambess.) Radlk. & Pitomba & Campos et al. 2015, Nascimento et al. 2013 \\
\hline \multicolumn{3}{|l|}{ SAPOTACEAE } \\
\hline Chrysophyllum arenarium Allemão & Cajazinha & Campos et al. 2015 \\
\hline Manilkara salzamanni (A.DC.) H.J. Lam & Maçaranduba & Gomes et al. 2020 \\
\hline Pouteria glomerata (Miq.) Radlk & Marmelada & Campos et al. 2015 \\
\hline Pouteria macrophylla (Lam.) Eyma & Taturubá & Chaves et al. 2015 \\
\hline $\begin{array}{l}\text { Sideroxylon obtusifolium (Roem. \& Schult.) } \\
\text { T.D. Penn. }\end{array}$ & Quixaba & Nascimento et al. 2011, Nascimento et al. 2013 \\
\hline
\end{tabular}

Fonte: Web of Science, Scopus.

\section{Potencialidades do Nordeste brasileiro}

As famílias botânicas com maior predominância em número de espécies e uso pelas populações são as Myrtaceae (15 espécies) e as Fabaceae (10 espécies), mesmo em diferentes estados do Nordeste. Especificamente a família Fabaceae foi indicada por Jacob et al. (2020) como um grupo estratégico para a alimentação. Os autores identificaram que esta família oferece quantidade elevada de proteína vegetal, valor nutricional importante, são resistentes, resilientes, enriquecem o solo com a fixação de nitrogênio e podem ser armazenadas por um tempo relativamente logo sem perda significativa de nutrientes relevantes.

A Figura 4 agrega a quantidade de citações de espécies contidas nas famílias botânicas, sendo representadas 
as que tiveram quantidade igual ou superior a quatro indicações nos trabalhos pesquisados para cada estado do nordeste. Myrtaceae possui representatividade elevada com ocorrência significativa, principalmente, no estado do Ceará. As famílias Anacardiaceae, Cactaceae e Arecaceae também apresentam porcentagens de utilização relevantes para este estudo.

O levantamento realizado apontou o importante diferencial alimentício das Cactáceas, com a utilização de praticamente todas as partes da planta na culinária, como por exemplo: o cladódio pode ser utilizado como hortaliça, para a fabricação de compotas, doces em pasta e o fruto (polpa) in natura pode ser consumido com mel e outros acompanhamentos (Chaves \& Barros 2015). A diversidade de preparos é ampla e podem ser inseridas como geradoras de renda para as comunidades em situação de vulnerabilidade (Chaves \& Barros 2015).

Nascimento et al. (2011) realizaram testes de composição centesimal em partes de plantas silvestres utilizadas e indicadas pelas comunidades e os cladódios de Pilocereus pachicladus F. Ritter. e Xiquexique gounellei (F. A. C. Weber) Lavor \& Calvente. Apesar de muito consumidos em comunidades rurais no Pernambuco, nesta análise, não se mostrou tão interessante para introdução na alimentação, pois apresenta baixo valor energético, características físico químicas não tão relevantes e grandes dificuldades relacionadas ao seu preparo e coleta.

A composição nutricional de um alimento é um dado relevante para a inserção de plantas silvestres na alimentação. Jacob \& Albuquerque (2020) indicam que essa é uma das lacunas que necessita ser preenchida pelos pesquisadores para que possam ser amplamente utilizadas, auxiliando na problemática de segurança alimentar. Análises de composição nutricional podem reforçar os usos já estabelecidos pelas comunidades e assegurar a ingestão em quantidades seguras e inserção em dietas sustentáveis.
Chaves et al. (2015) demonstraram por meio da análise de parâmetros nutricionais, físico-químicos, atividade antioxidante biológica e in vitro que seis espécies utilizadas por comunidades rurais do semiárido nordestino podem ser utilizadas como complementos alimentares e para exploração tecnológica. Os vegetais apresentaram alto teor de carboidratos e verificou-se que Hymenea martiana Hayne possui potencial para suprir a falta de culturas tradicionais como a do feijão, bastante utilizada pela comunidade rural estudada.

Vale ainda ressaltar, que as plantas alimentícias como, por exemplo, Zizipus joazeiro Mart., Cereus jamacuru DC. e Syagrus cearenses Noblick possuem possíveis aplicações no desenvolvimento de novos produtos, promovendo possibilidades de geração de renda para a população (Nascimento et al., 2011).

Bortolotto et al. (2017) no centro-oeste do Brasil, realizaram ações que envolveram comunidades rurais do cerrado e pantanal, universidades e organizações não governamentais, apresentaram resultados para a valorização dos conhecimentos da população e geração de renda, por meio de estratégias e novas alternativas econômicas, capacitando-os com cursos, treinamentos e oficinas de culinária evidenciando que se houver incentivos e projetos o uso e proteção da diversidade dos recursos naturais e saberes podem ser salvaguardados.

A percepção sobre o incipiente conhecimento da biodiversidade alimentícia, em contrapartida, favorece a abertura de diversas abordagens e olhares na consolidação de pesquisas nesta área. Há um leque de possibilidades alimentares e econômicas, características genéticas de interesse na melhoria das culturas convencionais, estudo de metabólitos secundários com eficácia para a defesa contra patógenos e pragas, a propagação das espécies mais adaptadas e melhor domesticadas, são algumas das possíveis linhas de estudo ainda negligenciadas.

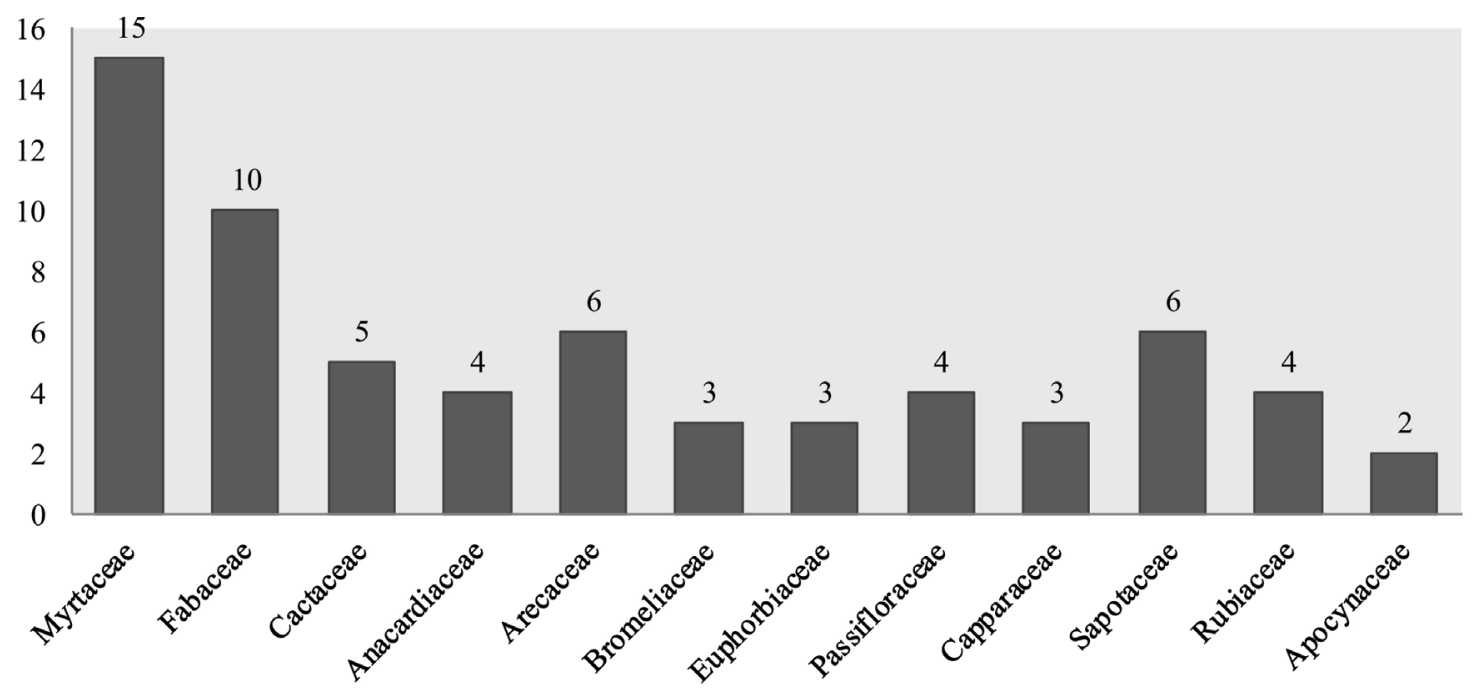

Figura 4. Famílias botânicas com maior quantidade de espécies citadas nos artigos científicos realizados no nordeste brasileiro 


\section{CONSIDERAÇÕES FINAIS}

Por meio do mapeamento de artigos científicos brasileiros sobre plantas alimentícias silvestres com foco no semiárido, este estudo demonstrou o potencial de crescimento dos trabalhos nesta área, revelando lacunas quanto à produção científica e identificação de PAS, levantamento e divulgação de dados sobre a biodiversidade do nordeste. Partindo dos artigos analisados, foram elencadas as principais famílias e espécies alimentícias indicadas por populações rurais em estudos etnobotânicos como recurso alimentar de complementação em tempos de escassez, como alimentos saborosos de alto valor energético ou ainda como gerador de renda.

A metodologia utilizada favoreceu o levantamento de estudos científicos realizados sobre plantas alimentícias silvestres desenvolvidas no mundo, Brasil e Nordeste. No entanto, os descritores selecionados podem não resgatar todas as pesquisas relevantes devido ao emprego de outros termos (como por exemplo: plantas comestíveis silvestres, plantas alimentícias selvagens etc.), mas que estão relacionadas aos utilizados neste estudo e não foram incluídas, pois a quantidade de artigos científicos identificados nas bases de dados foi menor quando comparadas às palavras-chave selecionadas.

Em síntese, ressalta-se que a escassa produção científica relacionada à temática (considerando os descritores utilizados e as bases de dados selecionadas neste estudo), exige urgente necessidade no desenvolvimento de mais pesquisas sobre o assunto. $\mathrm{O}$ aprofundamento do conhecimento e pesquisas futuras permitirá conhecer a composição química e nutricional, apontar novas espécies utilizadas pelas populações, a valorização dos modos de vida que legitimaram o uso e aplicações das plantas por várias gerações, visando ainda a perpetuação do saber para que não seja esquecido, assim como os recursos naturais têm perdido espaço para novas atividades humanas que degradam, exaurem e extinguem o equilíbrio dos ecossistemas.

\section{REFERÊNCIAS}

Akgul, A., Akgul, A., Senol, S. G., Yildirim, H. Secmen, O. \& Dogan, Y.2018. An ethnobotanical study in Midyat (Turkey), a city on the silk road where cultures meet. Journal of Ethnobiology and Ethnomedicine 14(12):1-19.

Albuquerque, U. P., do Nascimento, A. L. B., Chaves, L. S., Feitosa, I. S., Moura, J. M. B., Gonçalves, P. H. S., Silva, R. H., Silva, T. C. \& Ferreira Júnior, W. S. 2020. The chemical ecology approach to modern and early human use of medicinal plants. Chemoecology 30:89-102.

Andrade-Lima, D. de. 1981. The caatinga dominium. Revista Brasileira Botânica 4 (2):149-163.

Araújo, E. L., Castro, C. C. \& Albuquerque, U. P. 2007. Dynamics of Brazilian Caatinga - A Review Concerning the Plants, Environment and People. Functional Ecosystems and Communities 1(1):15-28.

Aworh, O. C. 2018. From lesser-known to super vegetables: the growing profile of African traditional leafy vegetables in promoting food security and wellness. Society of Chemical Industrial 98:3609-3613.

Barbieri, R. L., Gomes, J. C. C.; Alercia, A. \& Padulosi, S. 2014. Agricultural Biodiversity in Southern Brazil: Integrating Efforts for Conservation and Use of Neglected and Underutilized Species. Sustainability 6:741-757.

Borelli, T.; Hunter, D.; Powell, B.; Ulian, T.; Mattana, E.; Termote, C.; Pawera, L.; Beltrame, D.; Penafiel, D.; Tan, A.; Taylor, M. e Engels, J. 2020. Born to Eat Wild: An Integrated Conservation Approach to Secure Wild Food Plants for Food Security and Nutrition. Plants 9(1299):1-36.

Barros, I. O. \& Soares, A. A. 2013. Adaptações anatômicas em folhas de marmeleiro e velame da caatinga brasileira. Revista Ciência Agronômica 44(1):192-198.

Bortolotto, I. M., Damasceno-Júnior, G. A. \& Pott, A. 2018. Lista preliminar das plantas alimentícias. Iheringia Série Botânica 73: 101-116.

Bortolotto, I. M., Hiane, P., Ishii, I. H., Souza, P. R., Campos, R. P., Gomes, R. J. B., Farias, C. S., Leme, F. M., Arruda, R. C. O., Lima, L. B. \& Damasceno-Júnior, G. A.2017. A knowledge network to promote the use and valorization of wild food plants in the Pantanal and Cerrado, Brazil. Regional Environmental Change 17:1329-1341.

Bortolotto, I. M., Seleme, E. P., Araújo, I.P.P., Moura, S. S. \& Sartori, A. L. B. 2019. Conhecimento local sobre plantas alimentícias nativas no chaco brasileiro. Oecologia Australis 23(4):764-775.

Campos, L. Z. O., Albuquerque, U. P., Peroni, N. \&Araújo, E. L. 2015. Do socioeconomic characteristics explain the knowledge and use of native food plants in semiarid environments in Northeastern Brazil? Journal of Arid Environments 11(5):53-61.

Chaves, E. M. F., Siqueira, J. I. A., Morais, R. F. \& Barros, R. F. M. 2019. Conocimiento y uso de plantas silvestres alimentícias en comunidades campesinas de La región semiárida de Piauí, nordeste de Brasil. Ethnobotany Research and Applications 18(33):1:20.

Chaves, M. F. C., Morais, R. F. \& Barros, R. F. M. 2017. Práticas alimentares populares com uso de plantas silvestres: potencial para minimizar a insegurança nutricional no semiárido do Nordeste do Brasil. Gaia Scientia 11(2):287-313.

Chaves, M. F. C. \& Barros, R.F.M. 2015. Cactáceas: recurso alimentar emergencial no semiárido, Nordeste do Brasil. Gaia Scientia 9(2):129135.

Chaves, E. M. F., Silva, J. N., Lima, S., Albuquerque, U. P. \& Barros, R. F. M. 2015. Potential of wild food plants from the semi-arid region of northeast Brazil: chemical approach ethnoguided. Espacios 36(16):20.

Cruz, M. P., Medeiros, P., Sarmiento-Combariza, I., Peroni, N. \& Albuquerque, U. P. 2014. "I eat the manofê so it is not forgotten": local perceptions and consumption of native wild edible plants from seasonal dry forests in Brazil. Journal of Ethnobiology and Ethnomedicine 10(45):1- 11 .

Cruz, M. P., Peroni, N. \& Albuquerque, U. P. 2013. Knowledge, use and management of native wild edible plants from a seasonal dry forest (NE, Brazil). Journal of Ethnobiology and Ethnomedicine 9(79):1-10.

Cruz-Garcia, G.S., Caffi, C., Zans, M. E. C. \& Sanchez-Choy, J. 2018. Children's Knowledge of Wild Food Plants in the Forest-Agriculture Interface. Journal of Ethnobiology 3(2):205-222.

Cunha, J. A. S. \& Barros, R. F. M. 2018. Educação ambiental no ensino fundamental: revitalização do espaço escolar com o plantio de frutíferas e medicinais. Educação Ambiental Em Ação 65(17):1-12.

Farias, J. C., Miranda, G. D. R., Santos, M. H. B., Bonfim, B.L.S., Fonseca Filho, I. C., Franca, S. M., Barros, R. F. M. \& Silva, P.R. 2019. Medicinal flora cultivated in backyards of a community in Northeast Brazil. Ethnobotany research and applications 18:1-13.

Flora do Brasil 2020. Jardim Botânico do Rio de Janeiro. Disponível em: http://floradobrasil.jbrj.gov.br/ . Acesso em: 20 jan. 2021.

Gomes, D.L., Ferreira, R. P. S., Santos, E. M. C., Silva, R. R. V. \& Medeiros, P. M 2020. Local criteria for the selection of wild food plants for consumption and sale in Alagoas, Brazil. Ethnobiology and Conservation 9(10):1-15.

Guinand, Y. \& Lemessa, D. 2001. The potential of Indigenous wild foods. Diani, Kenya: USAID/OFDA. 26 p.

Hora, J. S. L., Da Silva, T. C. \& Nascimento, V. T. 2020. "É natural, é bom! São frutos que vem da natureza": representações locais sobre o consumo de plantas alimentícias silvestres em uma área rural do Brasil. Ethnoscientia 5(1):1-11. 
Ichikawa, M. 1980. The Utilization of Wild Food Plants by the Suiei Dorobo in Northern Kenya. The Journal of the Anthropological Society of Nippon 88(1):25-47.

Irvine, F. R. 1952. Supplementary and Emergency Food Plants of West Africa. Economic Botany 6(1):23-40.

Jacob, M. C. M., Medeiros, M. F. A. \&Albuquerque, U. P. 2020. Biodiverse food plants in the semiarid region of Brazil have unknown potential: A systematic review. Plos One 15(5):1-24.

Jacob, M. C. M. \& Albuquerque, U. P. 2020. Biodiverse food plants: Which gaps do we need to address to promote sustainable diets? Ethnobiology and Conservation 9(9):1- 6 .

Kinupp, V. F. \& Lorenzi, H. 2014. Plantas Alimentícias Não Convencionais (PANC) no Brasil: guia de identificação, aspectos nutricionais e receitas ilustradas. Instituto Plantarum de Estudos da Flora, São Paulo. 768 p.

Kinupp, V. F. \& Barros, I. B. I. 2008. Protein and mineral contents of native species, potential vegetables, and fruit. Ciência e Tecnologia de Alimentos 28(4):846-857.

Lins Neto, E. M. F. \& Albuquerque, U. P. 2018. Theories of Niche Construction and Optimal Foraging: weaknesses and virtues in understanding the early stages of domestication. Ethnobiology and Conservation 7(7):1-5.

Liporacci, H.S. N., Hanazaki, M., Ritter, M. R. \& Araújo, E. L. 2017. Where are the Brazilian ethnobotanical studies in the Atlantic Forest and Caatinga?. Rodriguésia 68(4):1225:1240.

Lulekal, E., Asfaw, Z., Kelbessa, E. \& Van Dame, P. 2011. Wild edible plants in Ethiopia: a review on their potential to combat food insecurity. Africa Focus 24(2):71-121.
Martinelli, G. \& Moraes, M. A. 2013. Livro vermelho da flora do Brasil. Andrea Jakobsson Estúdio Editorial, Jardim Botânico do Rio de Janeiro, Rio de Janeiro. 1100p.

Nascimento, V. T., Lucena, R. F. P., Maciel, M. I. S. \& Albuquerque, U. P. 2013. Knowledge and Use of Wild Food Plants in Areas of Dry Seasonal Forests in Brazil. Ecology of Food and Nutrition 52: $317-343$.

Nascimento, V. T., Moura, N. P., Vasconcelos, M. A. S., Maciel, M. I. S. \& Albuquerque, U. P. 2011. Chemical characterization of native wild plants of dry seasonal forests of the semi-arid region of northeastern Brazil. Food Research International 44: 2112-2119.

Nascimento, V. T., Vasconcelos, M. A. S., Maciel, M. I. S. \& Albuquerque, U. P.2012. Famine Foods of Brazil's Seasonal Dry Forests: Ethnobotanical and Nutritional Aspects. Economic Botany 66 (1): 23-34.

Oliveira, F. C. S. \& Barros, R. F. M. 2018. Aspectos sociodemográficos dos comerciantes de plantas medicinais da feira de Oeiras, Piauí. Educação ambiental em ação 63: 1-12.

Raven, P. H., Evert, R. F. \& Eichhorn, S. E. 2014. Biologia Vegetal. São Paulo: Editora Guanabara Koogan. 876 p.

Ritter, M. R., Silva T. C., Araújo, E. L. \& Albuquerque U. P. 2015. Bibliometric analysis of ethnobotanical research in Brazil (19882013). Acta Botanica Brasilica 29: 113-119.

Tomchinsky, B. \& Ming, L. C. 2019. As plantas comestíveis no Brasil dos séculos XVI e XVII segundo relatos de época. Rodriguésia 70: 1-16

Veasey, E. A., Piotto, F. A., Nascimento, W. F., Rodrigues, J. F., Mazette, T. F., Borges, A., Biguzzi, F. A., Santos, F. R. C., Sobierajski, G. R., Recchia, G. H., \& Mistro, J. C. 2011. Processos evolutivos e a origem das plantas cultivadas. Ciência Rural 41 (7): 1218 - 1228. 